\title{
Text Emotion Computing under Cognition Vision
}

\author{
Jingzhong Wang \& Lu Zhang \\ Information Engineering College, North China University of Technology \\ Beijing 100144, China \\ E-mail: jingzhongwang@163.com, elaine_ncut@163.com
}

\begin{abstract}
The emotion computation is an artificial intelligence popular research area. This article in view of text emotion computation research present situation, introduces in the emotion recognition the cognition linguistics and the psychology knowledge, attempts solves the existing problem from a new angle. Learned from experiment's result, the text emotion cognitive model's experiment effect is good.
\end{abstract}

Keywords: Emotion, Computing, Cognition vision

\section{Introduction}

With the development of Internet,the information which appears by text form are more and more frequent. It becomes us one kind of the most easily to gain and the richest interactive resources. Presently, however, the text emotion analysis aspect's research is little.Nowadays,with the uncausing development of natural language technology,people could extract emotion information from text through analyzing grammer strcture, Semantic information and emotion glossary methods etc. From the massive texts withdraws the emotion information which is contained in them has the broad application prospect in many aspects.For example, automated analysis the received mail emotion information,and give relevant emotion computing result before user reads. Through emotion analysis to the information in homepage, it may examine the homepage which contain violent emotion,realize homepage filtration and ensure the net's information security.

Along with natural language processing technology unceasing development, the text emotion computation has a richer method and reliable theory basis.

At present, the text emotion computation has the widespread application prospect in many domains. For example,speech synthesis, information security, intelligent robot, pattern recognition, personalized text, and analysis article emotion structure aspects and so on.

\section{Related research}

Regardless of the method of key word frequency statistics or the method based on semantic characteristic,the emotion glossary in the sentence is an important factor of emotion recognition. In the reality many sentences do not contain the emotion glossary, also has some sentences contain the complex grammar structure and the meaning, only join the corresponding grammar and the syntax analysis can it understand. Whereas the present majority Chinese syntaxes and the grammar analyzer's accuracy rate is low to the complex sentence analysis, introduce the glossary syntactic relation possibly to bring more noises. In view of the above situation, this article take the cognition linguistics and the mood psychology as a foundation, attempts from multiple perspectives and multi-dimension to solve text emotion computation's question, further enhances the accuracy rate of recognition.

\subsection{Mood, emotion and sentiment in psychology}

Meng Zhaolan in book"Mood Psychology" has made certain discrimination to mood,emotion and sentiment etc expressions. In this article, the emotion includes basic emotion which the humanity and the animal altogether have, and also includes the human some unique high-level emotions, it used American MIT laboratory professor R.Pcard's definition in her monograph "Emotion Computation" in 1997.

In the psychology research, many psychologists have elaborated the relations of mood and cognition. Liang Ning jian pointed out that person's psychological phenomenon divides into two parts which are both mutually connected and distinguished: Mechanism and personality. And the mechanism divides into the cognition process, mood emotion process and will process 


\subsection{Related knowledge of Text emotion recognition}

\subsubsection{The text emotion recognition particularity}

Text emotion recognition has similarity in emotion production mechanism with appearance ,sound and so on emotion recognition, but actually has the very big difference in the emotion manifestation and the concrete recognition methods. The text emotion recognition mainly concentrates in the semantic analysis to the context and the glossary. Because the environment where emotion occurs and the stimulating factor and so on are indicated through the language, rather than real stimulation through hears, saw and touches, therefore the language understanding veracity is the key of text emotion recognition, is also the most important part. To well understand the language meaning, from multi-dimensional, the three-dimensional angle explore syntax and the semantic structure, this article has introduced the cognition linguistics, the cognition pragmatics and the transference theory and so on related knowledge,enriches text emotion recognition method, expands its research view.

\subsubsection{Cognitive context}

The cognition pragmatics's foundation is connection theory,and that connection is a concept which depends on linguistic environment. Therefore, the connection theory's linguistic environment view has then become the cognition pragmatics research focal point, this linguistic environment is different with traditional linguistic environment concept,it is one kind of cognitive context view. The traditional context concept is jumbles together the latent context factor and the real context, had not explained how the context does affect the words understanding in the spoken language process, thus is unable to make the explanation for some questions.

The cognitive context is a psychological construction body, He Ziran thought that the cognitive context is composed by three kind of information: the logic information, encyclopedic information and words information. Xiong Xueliang thought that the cognitive context involves to the scene knowledge (concrete situation) the language context knowledge (work memory) and the background knowledge (knowledge structure), also includes the group consciousness which the social group altogether has, namely social token.

\section{Text emotion cognitive model}

This article uses the above mood theory, cognition psychology and cognition pragmatics and so on related knowledge foundation, synthesize text emotion recognition's characteristic, has given the emotion cognitive model. This model describe current sentence emotion through formula3.1

$\mathrm{E}=(\mathrm{S}, \mathrm{P}, \mathrm{CC}, \mathrm{TR})$

And $\mathrm{E}$ expressed the current sentence emotion, $\mathrm{S}$ represented the last sentence emotion, namely the current emotion foundation, $\mathrm{P}$ expressed the current emotion main body's charactor characteristic, $\mathrm{CC}$ represented the current sentence the cognitive context, TR represented the emotion migration rule, here is mainly refers to some emotion migration rule which counts from the corpus. The cognitive context is the essential part to define text emotion, therefore this article uses the cognition pragmatics related knowledge, describe cognitive context through formula 3.2

$\mathrm{CC}=(\mathrm{W}, \mathrm{K}, \mathrm{L})$

$\mathrm{W}$ expressed the current sentence glossary information, it is the generalized scope glossary, including emotion word, negative term, mark language and so on specific glossary, also includes syntax and grammar and so on information. $\mathrm{K}$ is cyclopedic knowledge, is refers to each kind of scheme emotion related in the text emotion recognition, $\mathrm{L}$ expresses the logic information, it is the ability which relates the glossary information and cyclopedic knowledge, the logic information decided how this concept contact and relates with what concept.

The text emotion cognitive model, from the emotion cognition's angle, synthesized each kind of possibility influence emotion experience factor, took each kind of factor into account, promoted text emotion recognition. For example, the glossary information supplied the sentence basic meaning, the glossary information's rich and accurate can activate the correct topographic model, thus inferred the appropriate emotion.

\section{Emotion computation model appraisal}

\subsection{Emotion computation model appraisal}

The text emotion appraisal did not have a quite authoritative evaluation method at present, however a good evaluation method may expose the main question, neglects these non-essence question .It is possible to guide the emotion analysis develop to a better direction, enhance labelling precise rate quickly. Regarding the sentence emotion appraisal, this article uses the following two appraisal method:

Appraisal method one: The sentence appraisal method takes the sentence as the division unit, in an article the labeled correct sentence number divides entire chapter article sentence number. As formula 4.1 


$$
P_{i}=\frac{1}{m} \sum_{k=1}^{m} f(k)
$$

If the kth sentence labelling is correct, the function value is 1 , otherwise the value is $0 . \mathrm{m}$ is the language total sentence number, $\mathrm{P}_{\mathrm{i}}$ is the language $\mathrm{i}$ emotion analysis accuracy rate. The result total accuracy weighs through the macro average and the micro average two methods. The macro average $\mathrm{P}_{\mathrm{ma}}$ namely is to compute each emotion category rate of accuracy's mean value, micro $\mathrm{P}_{\mathrm{mi}}$ is similar with the formula 4.1 , with all labelling correct sentence dividing total sentence

$$
P_{m a}=\frac{1}{n} \sum_{i}^{n} E i
$$

The formula 4.2 gave the computational method of macroscopic accuracy rate, $\mathrm{P}_{\mathrm{ma}}$ expressed the macroscopic rate of accuracy, $\mathrm{P}_{\mathrm{mi}}$ expressed that the ith kind of emotion's rate of accuracy, $\mathrm{n}$ expressed the emotion category number.

This evaluation method evaluated each sentence isolatedly, has shut off the sentence emotion connection relations, can only consider accuracy from the single sentence, but cannot effectively appraisal entire chapter article emotion labelling quality and the emotion continuity. For example possibly has following labelling result:

Correct labeling:tranquil, joyful, joyful, joyful, affection, tranquil, joyful, likes

Machine labeling1: tranquilly, joyful, joyful, joyful, affection, affection, affection, tranquil

Machine labelling2: tranquil, affection, joyful, affection, affection, affection, joyful, tranquil

We can see, although labels 1 and labels 2 has 5 labelling correct sentences, labeling result is bad in show emotion's continuous aspect. Therefore besides method one, this article has also used the appraisal method two, to make up and strengthen the emotion continuous appraisal.

Appraisal method 2: (union appraisal law): consult machine translation BLEU evaluation method, altogether the present glossary represents the target language by dual above the fluent degree. This article uses altogether the present multi-dimensional emotion the rate of accuracy which matches in standard labelling to weigh emotion labelling the continuity

$$
c_{d}=\frac{\sum_{i} \sum_{k=1}^{m i-d+1} f(k, d)}{\sum_{i} m^{i}}
$$

Formula $4.3 \mathrm{C}_{\mathrm{d}}$ expressed that $\mathrm{d}$ continual sentence's emotion labelling rate of accuracy, the function $\mathrm{f}(\mathrm{k}, \mathrm{d})$ expressed starting in the documents from $\mathrm{k}$ to take $\mathrm{d}$ labelling the emotion sentence and the correct emotion labelling match, if entire equal, is 1 , otherwise is 0 . $\mathrm{i}$ expressed that the ith documents, $\mathrm{m}$ expresses the ith documents total sentence .

\subsection{Experimental result and analysis}

This article takes periodical as the experiment materials, total 26757 sentences, nearly 800,000 characters. And training language materials 17457, the testing language materials 9300. Based on the above language materials, the experiment compared three method experimental results separately, the appraisal method has used the sentence appraisal and union appraisal method.

The method 1, the key word term frequency statistic

The method 2, emotion recognition based on the semantic characteristic

The method 3, emotion recognition based on cognition appraisal theory

Sentence appraisal's experimental result as shown in table 1, union appraisal method experimental result like table 2

May see from the table experimental result, the method 3 uses the text emotion cognitive model, the micro average rate of accuracy is $45 \%$. method two uses the condition stochastic territory the method labelling rate of accuracy is $43 \%$. Generally speaking, the text emotion cognitive model's experiment effect is good.

\section{Conclusions and future works}

Recognition methods based on the cognition introduced the cognition linguistics and the psychology knowledge into the emotion recognition, attempts to solve the existing problem from a new angle.

Future prime task: One, continues to expand the main body scale of the emotion glossary, enhance the automatic study the rate of accuracy. Second, further consummates the emotion corpus construction work, increases language materials labelling quantity, and will label the tool and the artificial revision automatically unifies, reduces the manual participation, speeds up the labelling speed. Third, consummates presently in the labelling speed and the rate of accuracy two aspects the some emotion labelling method, continues from the cognition linguistics and the mood 
psychology various exploration emotion recognition method, the rich emotion's topographic model, joins the individuality characteristic factor to the emotion the influence and so on.

\section{References}

Benjamin K Y, Kwong Y, Wong W L. (2005). Sentiment and content analysis of Chinese news co-verage. International Journal of Computer Processing of Oriental Languages, 2005, 18(2):171-183.

Lafferty J, McCallum A, Pereira F. (2001). Conditional random fields:probabilistic models for segmenting and labeling sequence data. International Conference on Machine Learning,Morgan Kaufmann, San Francisco, 2001:282-289.

Liu H, Lieberman H, Selker T. (2003). A model of textual affect sensing using real-world know-ledge. international Conference on Intelligent User Interfaces, Miami,Florida,2003:125-132.

Liu H, Selker T, Lieberman H. (2003). Visualizing the affective structure of a text document. Conference on Human Factors in Computing Systems, Lauderdale, Florida, 2003:740-741.

Ma C L, Pendinger H, Ishizuka M. (2005). Emotion estimation and reasoning based on affective textual interaction. International Conference of Affective Computing and Intelligent Interaction, Beijing, 2005:622-628.

Rosalind W P. (1997). Affective Computing. Cambridge: MIT Press, 1997.

Su W P. (2007). Personality and emotion-based high-level control of affective story chara-cters. IEEE Transactions on Visualization and Computer Graphics, 2007, 13(2):281-293.

Wang G J, Wang Z L, Li L X. (2005). An affective model of artificial psychology. IEEE International Conference on Communications, Circuits and Systems, Hong Kong, 2005:1061-1064.

Wang H,Prendinger H,Tgarashi T. (2004). Communicating emotions in online chat using physiolo-gical sensors and animated text. Conference on Human Factors in Computing Systems, Vienna, Austria, 2004:1171-1174.

Zhang Y, Li Z M, Ren F J et al. (2005). Semi-automatic emotion recognition from textual input based on the constructed emotion thesaurus. International Conference on Natural Language Processing and Knowledge Engineering, Wuhan, 2005:571-576.

Table 4.1 Three method experimental results

\begin{tabular}{llll}
\hline appraisal type & method 1 & method2 & method3 \\
\hline macro mean & 0.2846 & 0.3168 & 0.4057 \\
micro mean & 0.4130 & 0.4314 & 0.4462 \\
happy, & 0.5056 & 0.3534 & 0.4858 \\
anger & 0.1108 & 0.4744 & 0.8949 \\
sorrow & 0.1686 & 0.1202 & 0.1665 \\
fears & 0.1625 & 0.0092 & 0.2761 \\
wicked & 0.2409 & 0.4838 & 0.2941 \\
startled, & 0.1022 & 0.0895 & 0.1150 \\
tranquil & 0.6284 & 0.6872 & 0.5997 \\
\hline
\end{tabular}

Table 4.2 Results of union sentence evaluation

\begin{tabular}{lccc}
\hline accuracy rate & method 1 & method2 & method3 \\
\hline C2 & 0.1958 & 0.2423 & 0.2174 \\
C3 & 0.0967 & 0.1409 & 0.1128 \\
\hline
\end{tabular}

